\title{
Anaplastic Thyroid Carcinoma: A ceRNA Analysis Pointed to a Crosstalk between SOX2, TP53, and microRNA Biogenesis
}

\author{
Walter Arancio, ${ }^{1}$ Valeria Carina, ${ }^{1,2}$ Giuseppe Pizzolanti, ${ }^{1}$ Laura Tomasello, ${ }^{1}$ \\ Maria Pitrone, ${ }^{1}$ Concetta Baiamonte, ${ }^{1}$ Marco Calogero Amato, ${ }^{1}$ and Carla Giordano ${ }^{1}$ \\ ${ }^{1}$ Section of Cardio-Respiratory and Endocrine-Metabolic Diseases, Biomedical Department of Internal \\ and Specialist Medicine (Di.Bi.M.I.S.), University of Palermo, Piazza delle Cliniche 2, 90127 Palermo, Italy \\ ${ }^{2}$ Istituto Ortopedico Rizzoli (IOR), Section of Biology and Genetics, Department of Pathobiology \\ and Medical and Forensic Biotechnology (Di.Bi.Me.F.), University of Palermo, Via Divisi 83, 90100 Palermo, Italy
}

Correspondence should be addressed to Walter Arancio; walter.arancio@unipa.it

Received 30 May 2014; Revised 28 August 2014; Accepted 10 September 2014

Academic Editor: Giuliana Salvatore

Copyright (C) 2015 Walter Arancio et al. This is an open access article distributed under the Creative Commons Attribution License, which permits unrestricted use, distribution, and reproduction in any medium, provided the original work is properly cited.

\begin{abstract}
It has been suggested that cancer stem cells (CSC) may play a central role in oncogenesis, especially in undifferentiated tumours. Anaplastic thyroid carcinoma (ATC) has characteristics suggestive of a tumour enriched in CSC. Previous studies suggested that the stem cell factor SOX2 has a preeminent hierarchical role in determining the characteristics of stem cells in SW1736 ATC cell line. In detail, silencing SOX2 in SW1736 is able to suppress the expression of the stem markers analysed, strongly sensitizing the line to treatment with chemotherapeutic agents. Therefore, in order to further investigate the role of SOX2 in ATC, a competing endogenous RNA (ceRNA) analysis was conducted in order to isolate new functional partners of SOX2. Among the interactors, of particular interest are genes involved in the biogenesis of miRNAs (DICER1, RNASEN, and EIF2C2), in the control cell cycle (TP53, CCND1), and in mitochondrial activity (COX8A). The data suggest that stemness, microRNA biogenesis and functions, p53 regulatory network, cyclin D1, and cell cycle control, together with mitochondrial activity, might be coregulated.
\end{abstract}

\section{Introduction}

Anaplastic thyroid carcinoma (ATC) is a rare endocrine tumour. Its morphological features resemble undifferentiated neoplasm. Due to severe metastasis development and to the rapid fatal course, surgery is rarely performed. Radiotherapy and chemotherapy are also not very effective. It has been suggested that those standard therapies are ineffective because they are not able to efficiently target a subpopulation of ATC cells, called the cancer-initiating cells or cancer stem cells (CSCs). It has been proposed that CSCs possess stem-cell-like features, are at the core of the development of many tumours, especially undifferentiated ones like ATC, are responsible for the recurrence of the tumour and metastasis formation, and usually are very resistant to classical therapies.

Despite many controversies regarding the cancer stem cell model, it has the potential to drive the discovery of innovative treatments that may eradicate the very chemoresistant core of cancer [1]. In this connection, the CSC model is the sum of many hypotheses that have arisen to explain the most vexing aspects of cancer: metastasis, relapse, and therapeutic resistance [2]. In this perspective, CSC research holds out promise for improved treatment outcomes, in particular, as regards overcoming resistance to chemotherapy on solid tumours [1].

The most accepted CSC model makes use of a new paradigm of cellular differentiation, in which cancer cells can dedifferentiate toward more primitive, stem-like phenotypes [2]. The dedifferentiation seems to be highly heterogeneous, giving an explanation to the observed discontinuous behaviour of many cancers [2]. Alternatively, CSCs might arise from transformed stem cells in the stem niche $[1,2]$.

Similar to normal stem cells, CSCs have the ability both to self-renew and to give rise to differentiated tumour cells, are responsible for the organization of a tumour mass [3], and are tumorigenic when transplanted into an animal host [4]. CSCs 
TABLE 1: microRNAs that have been reported in the literature to regulate the main transcript from the SOX2 locus.

\begin{tabular}{|c|c|c|c|}
\hline hsa-let-7a & hsa-miR-125b-2* & hsa-miR-1914* & hsa-miR-30a* \\
\hline hsa-let-7a* & hsa-miR-126 & hsa-miR-1915 & hsa-miR-30b \\
\hline hsa-let-7b & hsa-miR-126* & hsa-miR-1915* & hsa-miR-30b* \\
\hline hsa-let-7b* & hsa-miR-134 & hsa-miR-200c & hsa-miR-30c \\
\hline hsa-let-7c & hsa-miR-137 & hsa-miR-200c* & hsa-miR-30c-1 ${ }^{*}$ \\
\hline hsa-let-7c* & hsa-miR-142-3p & hsa-miR-203 & hsa-miR-30c- $2^{*}$ \\
\hline hsa-let-7d & hsa-miR-143 & hsa-miR-204 & hsa-miR-30d \\
\hline hsa-let-7d* & hsa-miR-143* & hsa-miR-205 & hsa-miR-30d* \\
\hline hsa-let-7e & hsa-miR-145 & hsa-miR-206 & hsa-miR-30e \\
\hline hsa-let-7e* & hsa-miR-145* & hsa-miR-21 & hsa-miR-30e* \\
\hline hsa-let-7f & hsa-miR-155 & hsa-miR-21* & hsa-miR-452 \\
\hline hsa-let-7f-1 ${ }^{*}$ & hsa-miR-155* & hsa-miR-223 & hsa-miR- $452^{*}$ \\
\hline hsa-let-7f- $2^{*}$ & hsa-miR-17 & hsa-miR-223* & hsa-miR-9 \\
\hline hsa-let-7g & hsa-miR-17* & hsa-miR-296-3p & hsa-miR-9* \\
\hline hsa-let-7g* & hsa-miR-183 & hsa-miR-296-5p & hsa-miR-92a \\
\hline hsa-let-7i & hsa-miR-183* & hsa-miR-302a & hsa-miR-93 \\
\hline hsa-let-7i* & hsa-miR-1908 & hsa-miR-302a* & hsa-miR-93* \\
\hline hsa-miR-100 & hsa-miR-1909 & hsa-miR-302b & \\
\hline hsa-miR-100* & hsa-miR-1909* & hsa-miR-302b* & \\
\hline hsa-miR-106b & hsa-miR-1910 & hsa-miR-302c & \\
\hline hsa-miR-106b* & hsa-miR-1911 & hsa-miR-302c* & \\
\hline hsa-miR-125a-3p & hsa-miR-1911* & hsa-miR-302d & \\
\hline hsa-miR-125a-5p & hsa-miR-1912 & hsa-miR-302d* & \\
\hline hsa-miR-125b & hsa-miR-1913 & hsa-miR-302f & \\
\hline hsa-miR-125b-1* & hsa-miR-1914 & hsa-miR-30a & \\
\hline
\end{tabular}

have been identified in a wide range of human tumours [3]. At the molecular level, CSCs are usually enriched in cell surface markers such as CD44, CD24, and CD133, while Wnt/ $\beta$ catenin, Notch, and Hedgehog signalling pathways seem to have key roles in CSC properties [4]. Specific microRNA signatures have been identified in many CSCs [4] that seem to play a role in the epithelial-mesenchymal transition [4].

Regarding ATC, it has been hypothesized that the tumour initiates from transformed thyroid stem cells, rather than from differentiated thyrocytes undergoing a conventional multistep carcinogenesis model [5-7].

The rarity and rapid fatal nature of ATC has led to limited ex vivo studies. Here we describe an in vitro study on a well-validated ATC cell line: SW1736. The SW1736 cell line is characterized by a high percentage of population with stem cell-like properties and high expression of several stem markers (SOX2, OCT4, NANOG, C-MYC, SSEA4, and the ABCG2 transporter) [8]. Interestingly, SOX2 silencing downregulates in trans the expression of other stem cell markers and sensitizes ATC cells to treatment with classical chemotherapeutics such as cisplatin and doxorubicin [8]. This suggests that the stem cell factor SOX2 could have a preeminent hierarchical role in determining the characteristics of stem cells in SW1736 ATC cell line.

Therefore, in order to further investigate the role of SOX2 in ATC, a bioinformatic analysis of the functional network of SOX2 was performed. In detail, a competing endogenous RNA (ceRNA) analysis was conducted. This kind of analysis is able to predict genes functionally correlated with the bait gene rather than physically associated with it $[9,10]$. The ceRNA hypothesis is based on the rationale that RNA molecules can regulate one another via microRNAs $[9,10]$. ceRNAs are RNAs that share miRNA recognition elements, thereby regulating each other by influencing the available level of miRNA $[9,10]$. In the past, ceRNA analysis made it possible to isolate several genes and functional networks related to cancer development, ageing, and homeostasis [1119].

\section{Materials and Methods}

2.1. MirWALK Analysis. miRWalk is a comprehensive database that provides information on miRNA from humans, mice, and rats on their predicted as well as validated binding sites on their target genes. The validated targets module [20] hosts experimentally verified miRNA interaction with associated genes.

Using the miRWalk [20] data and embedded tools, we collected the microRNAs that have been reported in the literature to regulate the main transcript from the SOX2 locus (Table 1).

This set of miRNAs was inserted into the miRWalk analysis tool [20] to collect any human mRNA that has been reported to be regulated by them. Then the genes collected were organized in a hierarchical order for the number of validated microRNA hits (Table 2). The more microRNAs are 
TABLE 2: ceRNA organized in hierarchical order for the number of validated microRNA hits.

\begin{tabular}{|c|c|c|c|}
\hline Gene & Hits & Gene & Hits \\
\hline DICER1 & 35 & SLC27A4 & 10 \\
\hline TP53 & 26 & RUNX1 & 10 \\
\hline RNASEN & 22 & RRBP1 & 10 \\
\hline EIF2C2 & 22 & PAK3 & 10 \\
\hline COX8A & 22 & NFKB1 & 10 \\
\hline CCND1 & 22 & LIN28 & 10 \\
\hline MYC & 20 & KLF4 & 10 \\
\hline CDKN1A & 20 & FRAP1 & 10 \\
\hline BCL2 & 20 & EIF2C1 & 10 \\
\hline AKT1 & 19 & CREB1 & 10 \\
\hline PTEN & 18 & CDK6 & 10 \\
\hline CDKN2A & 18 & APC & 10 \\
\hline VEGFA & 16 & TWIST1 & 9 \\
\hline EGFR & 16 & SYNE1 & 9 \\
\hline TGFB1 & 14 & SIRT1 & 9 \\
\hline KRAS & 14 & PRDM1 & 9 \\
\hline JUN & 14 & MCL1 & 9 \\
\hline HMGA2 & 14 & HMOX1 & 9 \\
\hline ERBB2 & 14 & DNMT1 & 9 \\
\hline ZEB1 & 13 & DDX20 & 9 \\
\hline TLR4 & 13 & CKAP4 & 9 \\
\hline SSSCA1 & 13 & CDKN1C & 9 \\
\hline MET & 13 & BRCA1 & 9 \\
\hline TNF & 12 & ZNF828 & 8 \\
\hline SOCS1 & 12 & ТР63 & 8 \\
\hline PIK3CA & 12 & TIMM8A & 8 \\
\hline ESR1 & 12 & STMN1 & 8 \\
\hline DGCR8 & 12 & SCPEP1 & 8 \\
\hline СЕВРВ & 12 & ROS1 & 8 \\
\hline CD4 & 12 & PSAT1 & 8 \\
\hline TGFBR2 & 11 & PDCD4 & 8 \\
\hline STAT3 & 11 & MYCN & 8 \\
\hline PROM1 & 11 & MAPK3 & 8 \\
\hline NPC1 & 11 & JAK2 & 8 \\
\hline IL6 & 11 & IL1B & 8 \\
\hline EPHB2 & 11 & IFNG & 8 \\
\hline E2F3 & 11 & IFNA1 & 8 \\
\hline $\mathrm{E} 2 \mathrm{~F} 1$ & 11 & HMGA1 & 8 \\
\hline \multirow[t]{5}{*}{ CDKN1B } & 11 & GEMIN4 & 8 \\
\hline & & CTNNB1 & 8 \\
\hline & & CD19 & 8 \\
\hline & & BCL2L11 & 8 \\
\hline & & BAX & 8 \\
\hline
\end{tabular}

shared between the bait SOX2 gene and the candidate genes, the higher the possibility that the candidate gene transcripts can act as SOX2 ceRNAs. All analyses were updated to December 15, 2013.

2.2. GeneMANIA Analysis. Arbitrarily, the top 6 genes together with SOX2 were analysed using the GeneMANIA
[21] tool that helps to predict the functions of a set of genes and to predict in which gene ontology (GO) functions the set of genes might be involved (Figure 1) (Table 3). The GO functions reported are the ones with a false discovery rate $(\mathrm{FDR})<0.1$. All analyses were updated to December 15, 2013.

2.3. Cell Cultures. The SW1736, 8505C, C643, FRO, BCPAP, TPC-1, and WRO cell lines were cultured in Dulbecco's modified Eagle's medium high glucose medium supplemented with $10 \%$ fetal bovine serum and $5 \%$ glutamine. Cultures were maintained in $5 \%$ carbon dioxide at $37^{\circ} \mathrm{C}$ in a humidified incubator.

2.4. Small Interfering RNA (siRNA) Transfection. siRNA transfection in SW1736 cells was performed using INTERFERin transfection agent (Polyplus-Transfection, Illkirch, France), according to the manufacturer's instructions. Briefly, the transfection agent and the siRNA complex were added to the cells and incubated for 72 hours for RNA extraction and analysis. The final concentration of SOX2 siRNA was $100 \mathrm{nM}$. Each assay was performed in triplicate in at least three independent experiments. SOX2 was silenced using Stealth SiRNA SOX2 HSS144045 (Invitrogen, Milan, Italy). siCONTROL Stealth siRNA Negative Control was used as a control (Invitrogen, Milan).

2.5. SOX2 Coding Sequence Vector and Transient Transfection. The vector used was taken from Addgen (http://www.addgene.org/) (Plasmid 26817): pcDNA3.3_SOX2; and transformation into SW1736 cells was performed using Xfect transfection agent (Clontech Laboratories, Inc. A Takara Bio Company) according to the manufacturer's instructions. The transfection agent and plasmid were added to the cells and incubated for 72 hours for RNA extraction and analysis.

2.6. SOX2 $3^{\prime}$ Untranslated Region ( $\left.3^{\prime} U T R\right)$ Vector and Transfection. The vector was synthesized in service by Eurofins genomics (https://www.eurofinsgenomics.eu) using a pcDNA 3.1 backbone and a chemically synthesized $3^{\prime}$ UTR (as reported in http://mybioinfo.info/exon_display.php?tax_ id=9606\&gene_id=GeneID: 6657) (Table 4).

2.7. Reverse-Transcription PCR and Real-Time Quantitative $P C R$. Total RNA was extracted from cells using the RNeasy Mini Kit (Qiagen, Milan, Italy), including a digestion step with DNase I. RNA quantity and quality were assessed using the Nanodrop 2000 (Thermo Scientific, Wilmington, USA). The RNA extracted was reverse-transcribed with Random Hexamers (Applied Biosystems, Darmstadt, Germany) and Improm II Reverse Transcriptase (Promega Italia, Milan, Italy), according to the manufacturer's protocol. Primer pair sequences are reported in Table 5.

The reactions were performed as follows: $5^{\prime}$ at $94^{\circ} \mathrm{C}, 30$ cycles $\left(30^{\prime \prime}\right.$ at $94^{\circ} \mathrm{C}, 30^{\prime \prime}$ at $55^{\circ} \mathrm{C}, 30^{\prime \prime}$ at $\left.72^{\circ} \mathrm{C}\right), 5^{\prime}$ at $72^{\circ} \mathrm{C}$, and stocked at $4^{\circ} \mathrm{C}$. The only exception was the amplification of the SOX2 $3^{\prime} \mathrm{UTR}$, for which the following was done: $5^{\prime}$ at $94^{\circ} \mathrm{C}, 30$ cycles $\left(30^{\prime \prime}\right.$ at $94^{\circ} \mathrm{C}, 30^{\prime \prime}$ at $55^{\circ} \mathrm{C}, 90^{\prime \prime}$ at $\left.72^{\circ} \mathrm{C}\right), 5^{\prime}$ at $72^{\circ} \mathrm{C}$, and stocked at $4^{\circ} \mathrm{C}$. Expression was analyzed by realtime quantitative PCR (qRT-PCR) using Quantitect SYBR 


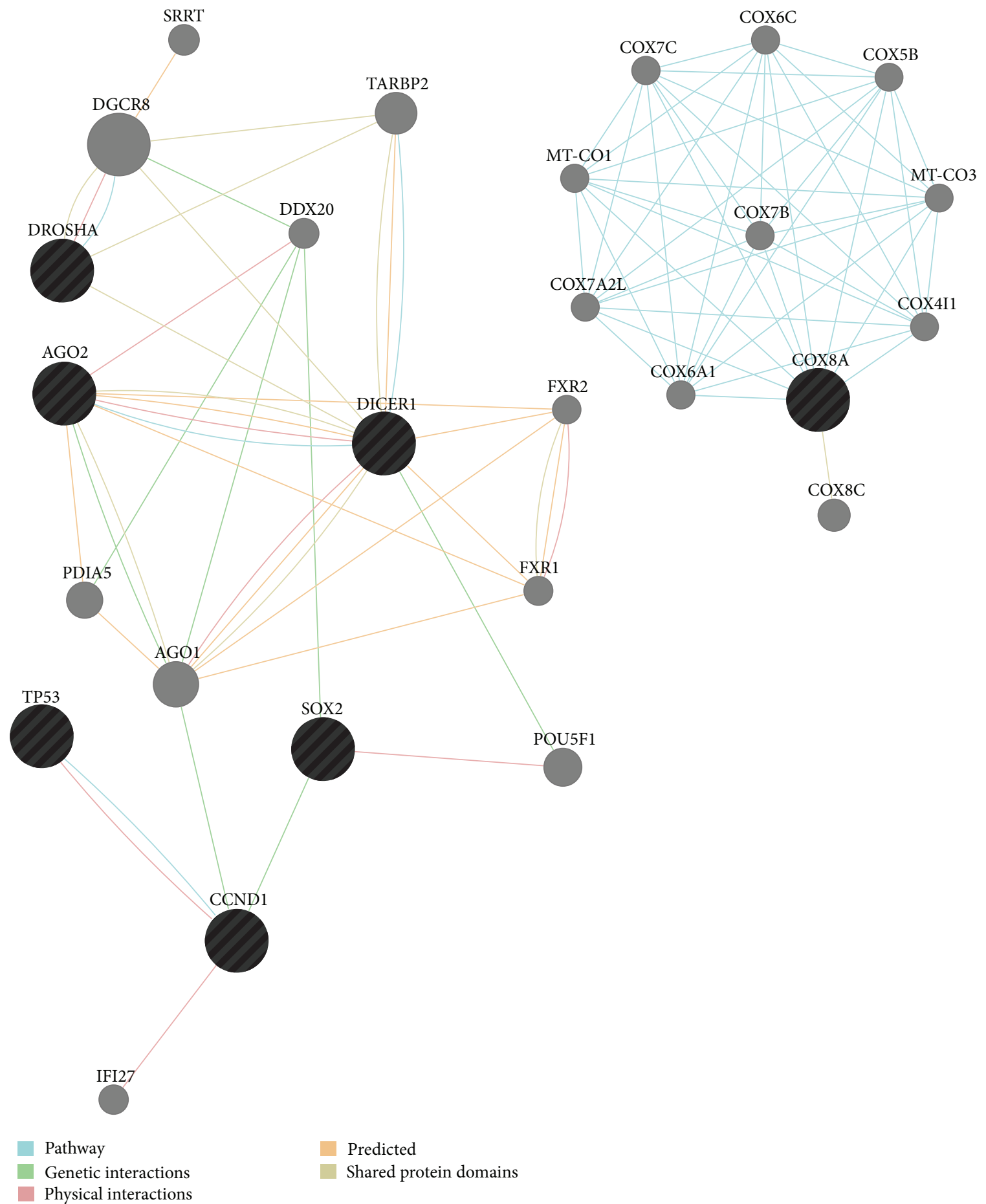

FIgURE 1: SOX2 ceRNA network by GeneMANIA.

Green PCR kit (Qiagen, Milan, Italy). All reactions were performed using a Rotor-gene Q Instrument (Qiagen, Milan, Italy). The data were analysed using the REST software [22].

2.8. Pool of Normal Thyroid Tissue. A pool of RNA from normal thyroid tissue specimens was used, as described in [23].
2.9. Limbal Stem Cell. A pool of RNA from limbal stem cells specimens was used, as described in [24].

2.10. Lymphocytes. Peripheral blood samples of a healthy volunteer were collected in tubes containing ethylenediaminetetraacetic acid (EDTA, $1 \mathrm{mg} / \mathrm{mL}$ ) after 8 hours' fasting. Lymphocytes were isolated by lympholyte (CEDARLANE, 
TABLE 3: Gene ontology of SOX2 ceRNA network by GeneMANIA.

\begin{tabular}{|c|c|c|}
\hline Function & $\begin{array}{l}\text { False } \\
\text { discovery } \\
\text { rate }\end{array}$ & Coverage \\
\hline Query genes & $\mathrm{n} / \mathrm{a}$ & $7 / 7$ \\
\hline Gene silencing by RNA & $3.14 e-15$ & $9 / 33$ \\
\hline Gene silencing & $5.01 e-13$ & $9 / 59$ \\
\hline Gene silencing by miRNA & $1.3 e-11$ & $7 / 25$ \\
\hline $\begin{array}{l}\text { Posttranscriptional gene silencing by } \\
\text { RNA }\end{array}$ & $1.92 e-11$ & $7 / 28$ \\
\hline Posttranscriptional gene silencing & $1.92 e-11$ & $7 / 28$ \\
\hline Regulation of gene expression, epigenetic & $5.32 e-9$ & $8 / 110$ \\
\hline $\begin{array}{l}\text { Production of miRNAs involved in gene } \\
\text { silencing by miRNA }\end{array}$ & $1.24 e-8$ & $5 / 13$ \\
\hline dsRNA fragmentation & $1.49 e-8$ & $5 / 14$ \\
\hline $\begin{array}{l}\text { Production of small RNA involved in } \\
\text { gene silencing by RNA }\end{array}$ & $1.49 e-8$ & $5 / 14$ \\
\hline Cellular response to dsRNA & $7.77 e-8$ & $5 / 19$ \\
\hline Response to dsRNA & $1.16 e-7$ & $5 / 21$ \\
\hline Respiratory electron transport chain & $1.16 e-7$ & $7 / 102$ \\
\hline Electron transport chain & $1.16 e-7$ & $7 / 103$ \\
\hline Cellular respiration & $7.68 e-7$ & $7 / 136$ \\
\hline Mitochondrial membrane & $3.2 e-6$ & $8 / 274$ \\
\hline ncRNA metabolic process & $5.44 e-6$ & $7 / 185$ \\
\hline Mitochondrial envelope & $5.44 e-6$ & $8 / 297$ \\
\hline Mitochondrial inner membrane & $7.4 e-6$ & $7 / 195$ \\
\hline Organelle inner membrane & $1.1 e-5$ & $7 / 208$ \\
\hline $\begin{array}{l}\text { Posttranscriptional regulation of gene } \\
\text { expression }\end{array}$ & $4.71 e-5$ & $7 / 259$ \\
\hline $\begin{array}{l}\text { Energy derivation by oxidation of organic } \\
\text { compounds }\end{array}$ & $7.46 e-5$ & $7 / 279$ \\
\hline $\begin{array}{l}\text { Cellular response to organic cyclic } \\
\text { compound }\end{array}$ & $2.23 e-4$ & $5 / 101$ \\
\hline ncRNA processing & $2.59 e-4$ & $5 / 105$ \\
\hline $\begin{array}{l}\text { Endonuclease activity, active with either } \\
\text { ribo- or deoxyribonucleic acids and } \\
\text { producing } 5^{\prime} \text {-phosphomonoesters }\end{array}$ & $6.97 e-4$ & $3 / 14$ \\
\hline Endoribonuclease activity & $1.25 e-3$ & $3 / 17$ \\
\hline Response to organic cyclic compound & $3.56 e-3$ & $5 / 183$ \\
\hline Stem cell maintenance & $1.09 e-2$ & $3 / 35$ \\
\hline Stem cell development & $1.25 e-2$ & $3 / 37$ \\
\hline Ribonuclease activity & $1.97 e-2$ & $3 / 44$ \\
\hline Endonuclease activity & $1.97 e-2$ & $3 / 44$ \\
\hline Stem cell differentiation & $4.17 e-2$ & $3 / 57$ \\
\hline Somatic stem cell maintenance & $5.63 e-2$ & $2 / 11$ \\
\hline Germplasm & $5.63 e-2$ & $2 / 11$ \\
\hline P granule & $5.63 e-2$ & $2 / 11$ \\
\hline Pole plasm & $5.63 e-2$ & $2 / 11$ \\
\hline Ribonucleoprotein granule & $6.92 e-2$ & $3 / 71$ \\
\hline Endodermal cell fate commitment & $7.54 e-2$ & $2 / 13$ \\
\hline
\end{tabular}

Burlington, Ontario, Canada), according to the manufacturer's instructions.
2.11. Statistical Analysis. We used the SPSS 13 software, Windows edition, for all our statistical analyses. Correlations were determined using Spearman's test (nonparametric equivalent for Pearson's test). $P<0.05$ was considered statistically significant.

\section{Results}

Previous data [8] suggested that the stem cell factor SOX2 possesses a preeminent hierarchical role in determining stemness characteristics in the SW1736 ATC cell line. With the final aim of investigating the role of SOX2 in ATC, a bioinformatic ceRNA analysis $[9,10]$ of the functional network of SOX2 was performed.

Using the miRWalk [20] data and embedded tools, we collected the microRNAs that have been reported in the literature to regulate the main transcript from the SOX2 locus (Table 1).

This set of miRNAs was inserted into the miRWalk analysis tool [20] to collect any human mRNA that has been reported to be regulated by them. Then the collected genes were organized in a hierarchical order for the number of validated microRNA hits (Table 2). The more microRNAs are shared between SOX2 and the candidate genes, the stronger the putative competitive effect that is at the core of the ceRNA hypothesis. The first six top level interactors were arbitrary selected for further analyses. The top level SOX2 interactors in this ceRNA analysis are DICER1, EIF2C2, and RNASEN, involved in miRNA biogenesis and functions [25]; the most studied antioncogene TP53, worthy of note because of its suggested role in stemness [26]; the nuclearcoded mitochondrial Cytochrome C Oxidase Subunit VIIIA COX8A [27]; and CCND1, the cyclin D coding gene [28].

Amongst the lesser interactors reported in Table 2, other genes might be worth studying in the future, especially for their involvement in oncogenesis (such as MYC, BCL2, PTEN, KRAS, JUN, and many others).

This study aimed to analyse whether a relationship might exist between the 6 top level interactors (DICER1, EIF2C2, RNASEN, TP53, COX8A, and CCND1) and SOX2 in the ATC cell line SW1736.

With this purpose in mind, the six interactors together with SOX2 were analysed by GeneMANIA software [21] to verify whether their putative network (Figure 1) might be enriched in some GO annotations. Unsurprisingly, the analysis revealed a statistically significant enrichment of miRNA-mediated, posttranscriptional gene silencing activities (Table 3).

Then we tried to establish in the SW1736 ATC cell line whether perturbations in the transcriptional state of SOX2 might alter in trans the transcriptional state of the ceRNAs identified. When we knocked down SOX2 transcripts via specific siRNA, all the ceRNAs were coherently downregulated in trans in RT-PCR analyses, as expected. The effect of the downregulation varied from one ceRNA to another but was always statistically significant [22] (Figure 2(a)) (Table 6). To further investigate whether the effect could be mediated by the impaired transcriptional factor activity of the protein coded by $S O X 2$, we evaluated whether the overexpression of 
TABLE 4: SOX2 3' untranslated region (3'UTR).

5'GGGCCGGACAGCGAACTGGAGGGGGGAGAAATTTTCAAAGAAAAACGAGGGAAATGGGAGGGGTGCAAAA GAGGAGAGTAAGAAACAGCATGGAGAAAACCCGGTACGCTCAAAAAGAAAAAGGAAAAAAAAAAATCCCATC ACCCACAGCAAATGACAGCTGCAAAAGAGAACACCAATCCCATCCACACTCACGCAAAAACCGCGATGCCGAC AAGAAAACTTTTATGAGAGAGATCCTGGACTTCTTTTTGGGGGACTATTTTTGTACAGAGAAAACCTGGGGA GGGTGGGGAGGGCGGGGGAATGGACCTTGTATAGATCTGGAGGAAAGAAAGCTACGAAAAACTTTTTAAAAG TTCTAGTGGTACGGTAGGAGCTTTGCAGGAAGTTTGCAAAAGTCTTTACCAATAATATTTAGAGCTAGTCTCC AAGCGACGAAAAAAATGTTTTAATATTTGCAAGCAACTTTTGTACAGTATTTATCGAGATAAACATGGCAAT CAAAATGTCCATTGTTTATAAGCTGAGAATTTGCCAATATTTTTCAAGGAGAGGCTTCTTGCTGAATTTTGA TTCTGCAGCTGAAATTTAGGACAGTTGCAAACGTGAAAAGAAGAAAATTATTCAAATTTGGACATTTTAATT GTTTAAAAATTGTACAAAAGGAAAAAATTAGAATAAGTACTGGCGAACCATCTCTGTGGTCTTGTTTAAAAA GGGCAAAAGTTTTAGACTGTACTAAATTTTATAACTTACTGTTAAAAGCAAAAATGGCCATGCAGGTTGACA CCGTTGGTAATTTATAATAGCTTTTGTTCGATCCCAACTTTCCATTTTGTTCAGATAAAAAAAACCATGAAAT TACTGTGTTTGAAATATTTTCTTATGGTTTGTAATATTTCTGTAAATTTATTGTGATATTTTAAGGTTTTCCC CCCTTTATTTTCCGTAGTTGTATTTTAAAAGATTCGGCTCTGTATTATTTGAATCAGTCTGCCGAGAATCCAT GTATATATTTGAACTAATATCATCCTTATAACAGGTACATTTTCAACTTAAGTTTTTACTCCATTATGCACAG TTTGAGATAAATAAATTTTTGAAATATGGACACTGAAA3'

TABLE 5: RT-PCR primer pairs.

\begin{tabular}{|c|c|c|}
\hline Gene & Forward primer $5^{\prime}>3^{\prime}$ & Reverse primer $5^{\prime}>3^{\prime}$ \\
\hline SOX2 CDS & GGAGACGGAGCTGAAGCCGC & GACGCGGTCCGGGCTGTTTT \\
\hline DICER1 & CTTTGCAACCCCTCAGCAT & TCATGAATTGCTTCTTGTTGC \\
\hline TP53 & ATCTACTGGGACGGAACAGC & GTGAGGCTCCCCTTTCTTG \\
\hline RNASEN & CACCGAGATCACAGTCATGG & TGTCTTCTCCTGTCGGGACT \\
\hline EIF2C2 & TCCACCTAGACCCGACTTTG & AACTCTCCTCGGGCACTTCT \\
\hline COX8A & TTACCTCCTGCTTCGTGACC & CACTCTGGCCTCCTGTAGGT \\
\hline CCND1 & ATGCCAACCTCCTCAACG & GGACCTCCTTCTGCACACAT \\
\hline SOX $23^{\prime} \mathrm{UTR}$ & CACCGGGCCGGACAGCGAACTGGAGGGGGG & TTTCAGTGTCCATATTTCAAAAATTTATTTATC \\
\hline$\beta$-ACTIN & GGACTTCGAGCAAGAGATGG & AGCACTGTGTTGGCGTACAG \\
\hline
\end{tabular}

the coding sequence of SOX 2 could have some in trans effects on ceRNAs. The coding sequence lacks the $3^{\prime}$ untranslated region ( $\left.3^{\prime} \mathrm{UTR}\right)$ that mainly bears the regulation mediated by miRNAs [29]. Our data indicate that no such effect occurs (Figure 2(b)) (Table 7), so the trans effect highlighted in (Figure 2(a)) is likely to be due to the endogenous miRNA competition, as in our hypothesis, rather than a classical interaction mediated by the proteic transcriptional factor SOX2. Finally, we investigated whether the overexpression of SOX2 3'UTR might have any effects in trans in the SW1736 ATC cell line. The effects were very modest, if present at all, but in line with the modulation that occurs during the competing events $[9,10]$. The most notable effect was the positive correlation, as expected, with the expression of EIF2C2 and SOX2 itself (Figure 2(c)) (Table 8).

The experiments previously described looked into the effects of perturbation of the expression of SOX2 on the expression of ceRNA genes in an ATC cell line. We then endeavoured to see whether any correlation might exist between the basal expression of SOX2 and the ceRNA genes in different specimens. In detail, we analysed by RT-PCR the relative expression of SOX2 and SOX2 ceRNAs compared to $\beta$-ACTIN expression in SW1736 ATC cell line, in $8505 \mathrm{C}$ ATC cell line, C643 ATC cell line, FRO ATC cell line,
BCPAP papillary thyroid carcinoma (PTC) cell line, TPC1 PTC cell line, WRO follicular thyroid carcinoma, and a pool of normal thyroid tissues present in the laboratory from previous experiments [23], a pool of limbal stem cells [24], and isolated lymphocytes from a male donor of 36 years old (see Supplementary Table 1 in Supplementary Material available online at http://dx.doi.org/10.1155/2014/439370). Interestingly, this analysis suggested a correlation in the basal expression of DICER1, RNASEN, and EIF2C2 (Table 9), as can be expected of genes whose functions are strictly coregulated in the biogenesis and function of microRNA, but surprisingly their basal expression seemed also to be somehow related to the basal expression of TP53 (Table 9), suggesting interesting scenarios that will be discussed shortly.

\section{Discussion}

The ceRNA bioinformatics analysis pointed to a list of genes that could be functionally coregulated with the stem transcriptional factor SOX 2 by a crosstalk mediated by several miRNAs. In our analysis, we used interactions reported in the literature instead of bioinformatically predicted ones as done in the past [17-19]. This approach makes it possible to harvest 


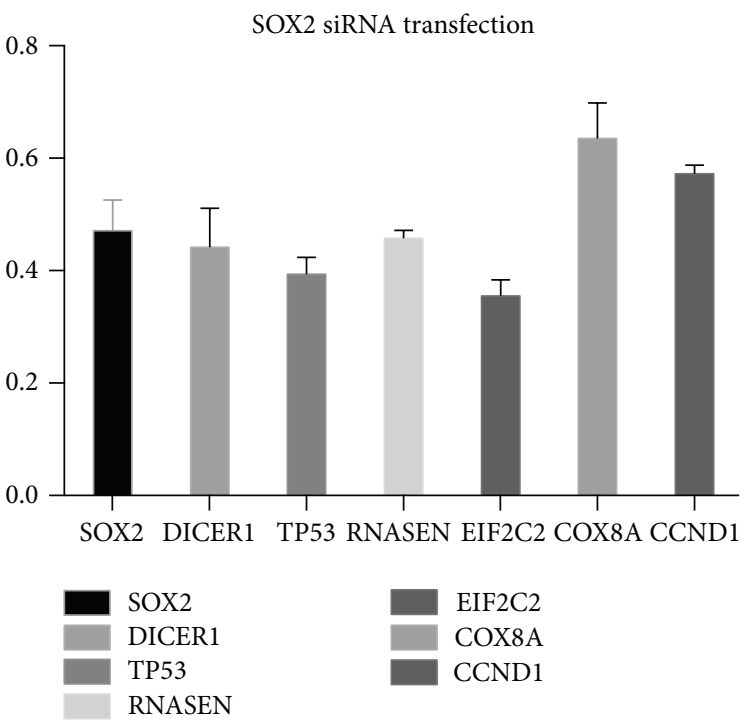

(a)

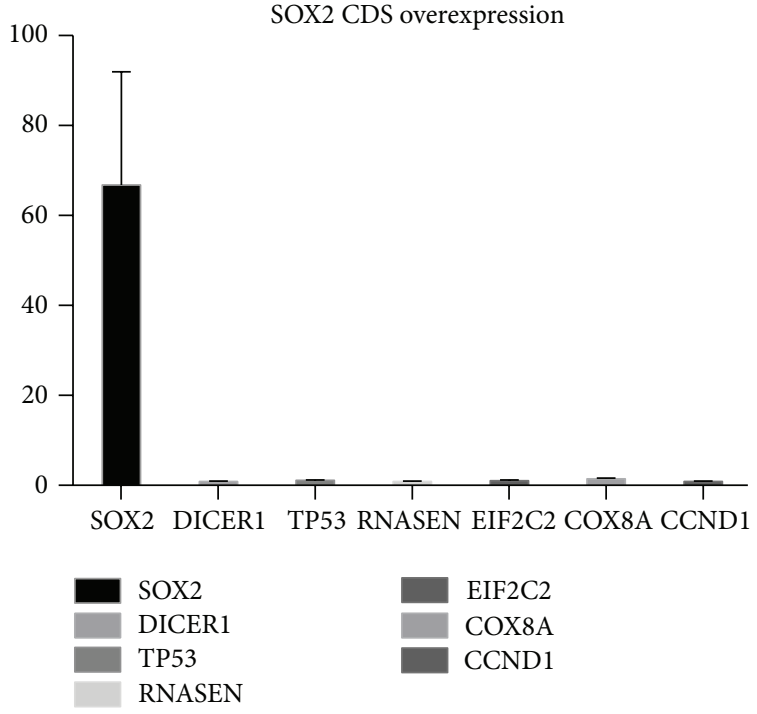

(b)

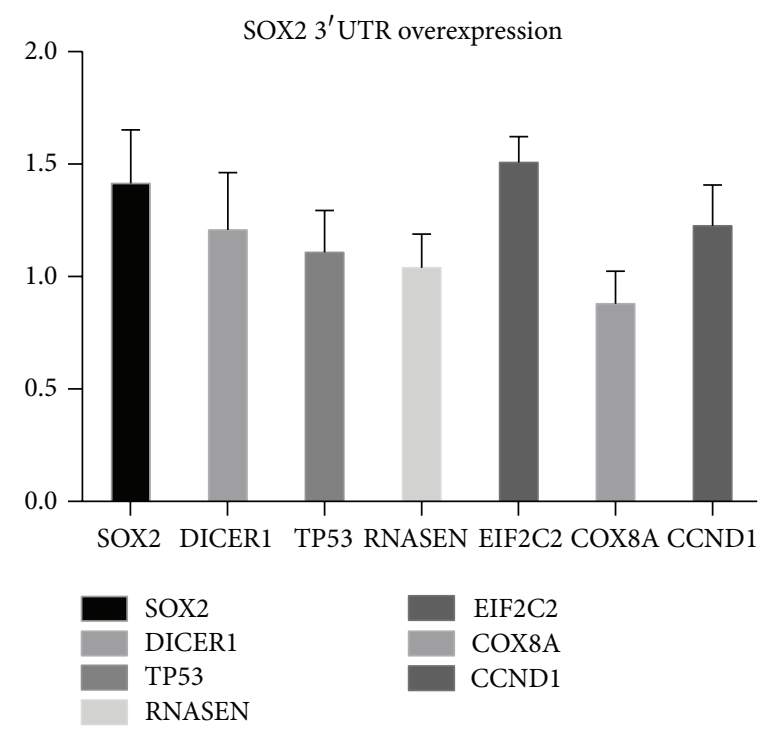

(c)

FIgURE 2: Example of SOX2 and SOX2 ceRNAs levels of transcription quantified by RT-PCR compared to controls and normalized against $\beta$-actin expression in SW1736 ATC cell line. Whiskers represent the standard errors. (a) Analysis of SOX2 silencing. (b) Analysis of SOX2 coding sequence overexpression. (c) Analysis of SOX2 $3^{\prime}$ UTR overexpression.

TABLE 6: Example of REST analysis on SOX2 and SOX2 ceRNAs levels of transcription quantified by RT-PCR compared to controls and normalized against $\beta$-actin expression in SW1736 ATC cell line after SOX2 silencing. $\mathrm{P}(\mathrm{H} 1)$ is the probability of the alternative hypothesis that the difference between sample and control groups is due only to chance.

\begin{tabular}{lcccccc}
\hline Gene & Reaction efficiency & Expression & Std. error & 95\% C.I. & P(H1) & Result \\
\hline SOX2 & 0.6375 & $\mathbf{0 . 4 7 1}$ & $0.416-0.538$ & $0.384-0.580$ & 0.000 & DOWN \\
DICER1 & 0.6125 & $\mathbf{0 . 4 4 2}$ & $0.373-0.523$ & $0.366-0.533$ & 0.000 & DOWN \\
TP53 & 0.7025 & $\mathbf{0 . 3 9 4}$ & $0.364-0.427$ & $0.348-0.445$ & 0.170 & DOWN \\
RNASEN & 0.73 & $\mathbf{0 . 4 5 8}$ & $0.444-0.472$ & $0.444-0.472$ & 0.000 & DOWN \\
EIF2C2 & 0.7475 & $\mathbf{0 . 3 5 5}$ & $0.326-0.386$ & $0.320-0.394$ & 0.000 & DOWN \\
COX8A & 0.69 & $\mathbf{0 . 6 3 5}$ & $0.572-0.705$ & $0.560-0.720$ & 0.000 & DOWN \\
CCND1 & 0.6875 & $\mathbf{0 . 5 7 2}$ & $0.556-0.588$ & $0.556-0.588$ & 0.000 & DOWN \\
\hline
\end{tabular}


TABLE 7: Example of REST analysis on SOX2 and SOX2 ceRNAs levels of transcription quantified by RT-PCR compared to controls and normalized against $\beta$-actin expression in SW1736 ATC cell line after SOX2 coding sequence (CDS) overexpression. $\mathrm{P}(\mathrm{H} 1)$ is the probability of the alternative hypothesis that the difference between sample and control groups is due only to chance.

\begin{tabular}{lcccccc}
\hline Gene & Reaction efficiency & Expression & Std. error & 95\% C.I. & P(H1) & Result \\
\hline SOX2 & 0.63 & $\mathbf{6 6 . 7 8 3}$ & $41.627-98.592$ & $37.461-120.196$ & 0.000 & UP \\
DICER1 & 0.67 & $\mathbf{0 . 8 4 7}$ & $0.750-0.981$ & $0.677-1.034$ & 0.131 & 0.136 \\
TP53 & 0.8175 & $\mathbf{1 . 0 9 9}$ & $1.000-1.198$ & $0.953-1.261$ & 0.131 \\
RNASEN & 0.7475 & $\mathbf{0 . 8 4 0}$ & $0.707-1.001$ & $0.672-1.059$ & 0.854 \\
EIF2C2 & 0.7725 & $\mathbf{1 . 0 1 5}$ & $0.829-1.246$ & $0.788-1.320$ & 0.000 \\
COX8A & 0.765 & $\mathbf{1 . 4 3 0}$ & $1.197-1.699$ & $1.136-1.788$ & UP \\
CCND1 & 0.755 & $\mathbf{0 . 8 6 1}$ & $0.763-0.947$ & $0.721-1.056$ & 0.080 \\
\hline
\end{tabular}

TABLE 8: Example of REST analysis on SOX2 and SOX2 ceRNAs levels of transcription quantified by RT-PCR compared to controls and normalized against $\beta$-actin expression in SW1736 ATC cell line after SOX2 $3^{\prime} \mathrm{UTR}$ overexpression. $\mathrm{P}(\mathrm{H} 1)$ is the probability of the alternative hypothesis that the difference between sample and control groups is due only to chance.

\begin{tabular}{|c|c|c|c|c|c|c|}
\hline Gene & Reaction efficiency & Expression & Std. error & 95\% C.I. & $\mathrm{P}(\mathrm{H} 1)$ & Result \\
\hline SOX2 & 0.655 & 1.414 & $1.176-1.710$ & $1.102-1.818$ & 0.000 & UP \\
\hline DICER1 & 0.7025 & 1.207 & $0.952-1.536$ & $0.912-1.600$ & 0.680 & \\
\hline TP53 & 0.77 & 1.105 & $0.918-1.332$ & $0.881-1.386$ & 0.661 & \\
\hline RNASEN & 0.75 & 1.039 & $0.889-1.222$ & $0.833-1.299$ & 0.830 & \\
\hline EIF2C2 & 0.765 & 1.508 & $1.393-1.641$ & $1.314-1.733$ & 0.169 & UP \\
\hline COX8A & 0.785 & 0.879 & $0.733-1.068$ & $0.669-1.160$ & 0.680 & \\
\hline CCND1 & 0.7425 & 1.226 & $1.045-1.462$ & $0.937-1.611$ & 0.341 & \\
\hline SOX $23^{\prime} \mathrm{UTR}$ & 0.64 & 3.092 & $2.577-3.762$ & $2.330-4.119$ & 0.000 & UP \\
\hline
\end{tabular}

TABLE 9: Spearman two-tailed test correlations between basal gene expressions (as reported in Supplementary Table 1) among SW1736, 8505C, C643, FRO, BCPAP, TPC-1, WRO, normal thyroid pool, limbal stem cells, and lymphocytes.

\begin{tabular}{|c|c|c|c|c|c|c|c|}
\hline & SOX2 & DICER1 & TP53 & RNASEN & EIF2C2 & COX8A & CCND1 \\
\hline \multicolumn{8}{|l|}{ SOX2 } \\
\hline Rho & 1 & 0.152 & 0.2 & -0.176 & 0.042 & 0.321 & -0.467 \\
\hline$P$ & & 0.676 & 0.580 & 0.627 & 0.907 & 0.365 & 0.174 \\
\hline \multicolumn{8}{|l|}{ DICER1 } \\
\hline Rho & 0.152 & 1 & 0.939 & 0.709 & 0.952 & 0.030 & -0.067 \\
\hline$P$ & 0.676 & - & $<0.001$ & 0.022 & $<0.001$ & 0.934 & 0.855 \\
\hline \multicolumn{8}{|l|}{ TP53 } \\
\hline Rho & 0.200 & 0.939 & 1 & 0.770 & 0.939 & -0.115 & -0.042 \\
\hline$P$ & 0.580 & $<0.001$ & - & 0.009 & $<0.001$ & 0.751 & 0.907 \\
\hline \multicolumn{8}{|c|}{ RNASEN } \\
\hline Rho & -0.176 & 0.709 & 0.770 & 1 & 0.842 & -0.382 & 0.261 \\
\hline$P$ & 0.627 & 0.022 & 0.009 & - & 0.002 & 0.276 & 0.467 \\
\hline \multicolumn{8}{|l|}{ EIF2C2 } \\
\hline Rho & 0.042 & 0.952 & 0.939 & 0.842 & 1 & -0.127 & 0.067 \\
\hline$P$ & 0.907 & $<0.001$ & $<0.001$ & 0.002 & - & 0.726 & 0.855 \\
\hline \multicolumn{8}{|l|}{ COX8A } \\
\hline Rho & 0.321 & 0.030 & -0.115 & -0.382 & -0.127 & 1 & -0.127 \\
\hline$P$ & 0.365 & 0.934 & 0.751 & 0.276 & 0.726 & - & 0.726 \\
\hline \multicolumn{8}{|l|}{ CCND1 } \\
\hline Rho & -0.467 & -0.067 & -0.042 & 0.261 & 0.067 & -0.127 & 1 \\
\hline$P$ & 0.174 & 0.855 & 0.907 & 0.467 & 0.855 & 0.726 & - \\
\hline
\end{tabular}


more solid and reliable data, though it is easier to collect genes that have been previously analysed.

Our experiments were pursued in an ATC cell line that has previously been demonstrated to constitutively express SOX2 that functionally possesses a preeminent hierarchical role on many other stem cell factors [8], suggesting a leading role in the maintenance of the stemness feature in this cell line. ATC represents a very good candidate for a cancer highly enriched in CSCs, which probably are at the core of its unfavourable outcome [1-7]. For these reasons, it is both important to understand the regulatory network that underlies the functions of SOX2 in ATC, and at the same time an ATC cell line is a very good candidate for studying the SOX2 network.

Looking at the cross-regulation between SOX2 and the most probable ceRNAs that we isolated, many if not all the ceRNAs analysed seem to be responsive to alterations in the transcriptional state of SOX2 transcripts, independently of the coded protein, suggesting a regulatory network strictly based on noncoding-RNAs (ncRNAs). The most striking evidence is the effect of siRNA-mediated silencing on $S O X 2$, where all the ceRNAs are accordingly downregulated (Figure 1(a)) (Table 6). By contrast, the overexpression of the SOX2 CDS alone seems to have almost no effect at all (Figure 1(b)) (Table 7). In contrast, the overexpression of the $3^{\prime}$ UTR of the SOX2 transcripts seems to have an upregulation effect in trans, even if not to a great degree (Figure 1(c)) (Table 8). The $3^{\prime} U T R$ of transcripts is the portion of messengers that is likely to bear the vast majority of regulation mediated by microRNAs [30]. The data reported here are consistent with our hypothesis, so it is reasonable to point to the ceRNAs isolated as potential functional interactors with SOX2, at least in the SW1736 ATC cell line.

The interactors isolated pointed to a central role of microRNA biogenesis and functions in SOX2 activities (Table 3) and hence in stemness, as other authors have recently suggested [29]. Here we report that probably the transcription of SOX2 stem factors and of Dicer (DICER1), Ago2 (EIF2C2), and Drosha (RNASEN) is coregulated by a microRNA network. In detail, Drosha is a RNA-specific endoribonuclease that is involved in the initial nuclear step of microRNA biogenesis. Dicer is a cytoplasmic endoribonuclease that plays a central role in the production of short interfering RNAs (siRNA) and mature microRNAs. SiRNAs and microRNAs serve as a guide to directing the RNA-induced silencing complex (RISC) to complementary RNAs to degrade them or prevent their translation. Ago2 is the essential proteic core of the RISC complex. Overall, the miRNA pathway is a means to specifically regulate the expression of target genes that seem to directly and indirectly affect tumorigenesis [31].

The SOX2 ceRNA TP53 gene codes for p53, one of the most studied genes in relation to cancer development [32]. It is also often mutated in ATC towards a nonfunctional form [33]. Nevertheless, even a mutated form, if transcribed, can still exert its regulatory functions via its transcript (e.g., its $3^{\prime} U T R$ ). In this perspective, homozygous deletion of the locus or full silencing of the gene perturbs the network differently from a null mutation, which still permits transcription from the locus. In the authors' opinion, this distinction is often not taken into account. It is interesting to note that some authors have suggested a role of p53 in homoeostasis of the stem niche [26] and in microRNA biogenesis [34], setting it at a crossroads between cancer, stemness, and microRNA biogenesis and functions. Our data are in support of this interpretation, all the more so because the basal transcription of TP53 seems to be correlated with the basal transcription of DICER1, EIF2C2, and RNASEN in the specimens that we analysed, many of them from ATC and other thyroid cell lines (Table 9).

The SOX2 ceRNA CCND1 codes for cyclin D1, the regulatory subunit that promotes G1/S cell-cycle progression and is involved in oncogenesis. It has been reported that cyclin D1 induces Dicer expression in vitro and in vivo and vice versa and their expression significantly correlates each other (at least in some subtypes of human breast cancer). It has been suggested that cyclin D1 induction of Dicer coordinates microRNA biogenesis $[28,35]$. Our data are in line with the previous results and add a new level of possible crosstalk between DICER1 and CCND1, suggesting novel actors in the network previously isolated, such as SOX2 or TP53. It is likely that cross-regulation between cyclin D1 and Dicer might occur in other cancers, especially in ATC, which are enriched in SOX2 producing cells [8], which we suggest is part of the network.

The role of COX8A is more difficult to appraise. The protein encoded by this gene is the terminal enzyme of the respiratory chain that leads to the production of the electrochemical gradient across the inner mitochondrial membrane. Recent discoveries suggest central roles of mitochondria in the maintenance of pluripotency, differentiation, reprogramming, and ageing [36]. Our data suggest possible crosstalk between a crucial nuclear coded mitochondrial factor and cell fate determinants such as SOX2 and TP53.

\section{Conclusions}

The SW1736 ATC cell line was used to investigate functional SOX2 interactors isolated by a novel bioinformatics analysis. Because SOX2 seems to have a central role in the maintenance of stem features in the SW1736 ATC cell line, the interactors are likely to play a role in stemness regulation.

The analysis pointed to DICER1, EIF2C2, RNASEN, TP53, COX8A, and CCND1 genes, suggesting that stemness, microRNA biogenesis and functions, p53 regulatory network, cyclin D1, and cell cycle control, together with mitochondrial activity, might be coregulated as a whole in their functions. Our data and previous data from the literature indicate that those functions are strictly interlinked and that deregulation of them might lead to cancer transformation, especially in cancers such as ATC that possess an undifferentiated nature suggestive of cancer stem cell enrichment.

\section{Conflict of Interests}

The authors declare that there is no conflict of interests regarding the publication of this paper. 


\section{Acknowledgments}

The authors would like to thank Dr. S.I. Genovese and D. Gailor for the help and advice given and Giordano Bruno, Nikola Tesla, and Barbara McClintock for their inspiring vision. This work has been partially funded by PON01_00829.

\section{References}

[1] M. L. O'Connor, D. Xiang, S. Shigdar et al., "Cancer stem cells: a contentious hypothesis now moving forward," Cancer Letters, vol. 344, no. 2, pp. 180-187, 2014.

[2] W. M. ElShamy and R. J. Duhé, "Overview: cellular plasticity, cancer stem cells and metastasis," Cancer Letters, vol. 341, no. 1, pp. 2-8, 2013.

[3] E. Sugihara and H. Saya, "Complexity of cancer stem cells", International Journal of Cancer, vol. 132, no. 6, pp. 1249-1259, 2013.

[4] Z. Yu, T. G. Pestell, M. P. Lisanti, and R. G. Pestell, "Cancer stem cells," International Journal of Biochemistry and Cell Biology, vol. 44, no. 12, pp. 2144-2151, 2012.

[5] R. Lloyd, Z. Guo, and H. Hardin, "Cancer stem-like cells and thyroid cancer," Endocrine-Related Cancer, 2014.

[6] J. Y. Yun, Y. A. Kim, J.-Y. Choe et al., "Expression of cancer stem cell markers is more frequent in anaplastic thyroid carcinoma compared to papillary thyroid carcinoma and is related to adverse clinical outcome," Journal of Clinical Pathology, vol. 67, no. 2, pp. 125-133, 2014.

[7] H. Hardin, C. Montemayor-Garcia, and R. V. Lloyd, “Thyroid cancer stem-like cells and epithelial-mesenchymal transition in thyroid cancers," Human Pathology, vol. 44, no. 9, pp. 1707-1713, 2013.

[8] V. Carina, G. Zito, G. Pizzolanti et al., "Multiple pluripotent stem cell markers in human anaplastic thyroid cancer: the putative upstream role of SOX2," Thyroid, vol. 23, no. 7, pp. 829837, 2013.

[9] L. Salmena, L. Poliseno, Y. Tay, L. Kats, and P. P. Pandolfi, "A ceRNA hypothesis: the rosetta stone of a hidden RNA language?" Cell, vol. 146, no. 3, pp. 353-358, 2011.

[10] R. Sen, S. Ghosal, S. Das, S. Balti, and J. Chakrabarti, "Competing endogenous RNA: the key to posttranscriptional regulation," The Scientific World Journal, vol. 2014, Article ID 896206, 6 pages, 2014.

[11] F. A. Karreth and P. P. Pandolfi, "CeRNA cross-talk in cancer: when ce-bling rivalries go awry," Cancer Discovery, vol. 3, no. 10, pp. 1113-1121, 2013.

[12] A. de Giorgioa, J. Krell, V. Harding, J. Stebbing, and L. Castellano, "Emerging roles of competing endogenous RNAs in cancer: insights from the regulation of PTEN," Molecular \& Cellular Biology, vol. 33, no. 20, pp. 3976-3982, 2013.

[13] X. Su, J. Xing, Z. Wang, L. Chen, M. Cui, and B. Jiang, "MicroRNAs and ceRNAs: RNA networks in pathogenesis of cancer," Chinese Journal of Cancer Research, vol. 25, no. 2, pp. 235-239, 2013.

[14] X. Song, G. Cao, L. Jing et al., "Analysing the relationship between lncRNA and protein-coding gene and the role of lncRNA as ceRNA in pulmonary fibrosis," Journal of Cellular and Molecular Medicine, vol. 18, no. 6, pp. 991-1003, 2014.

[15] X. Guo, M. Lin, S. Rockowitz, H. M. Lachman, and D. Zheng, "Characterization of human pseudogene-derived non-coding RNAs for functional potential," PLoS ONE, vol. 9, no. 4, Article ID e93972, 2014.
[16] M. S. Kumar, E. Armenteros-Monterroso, P. East et al., "HMGA2 functions as a competing endogenous RNA to promote lung cancer progression," Nature, vol. 505, no. 7482, pp. 212-217, 2014.

[17] W. Arancio, G. Pizzolanti, S. I. Genovese, C. Baiamonte, and C. Giordano, "Competing endogenous RNA and interactome bioinformatic analyses on human telomerase," Rejuvenation Research, vol. 17, no. 2, pp. 161-167, 2014.

[18] W. Arancio, C. Giordano, and G. Pizzolanti, "A ceRNA analysis on LMNA gene focusing on the Hutchinson-Gilford progeria syndrome," Journal of Clinical Bioinformatics, vol. 3, no. 1, article 2, 2013.

[19] W. Arancio, "A bioinformatics analysis of lamin-a regulatory network: a perspective on epigenetic involvement in hutchinson-gilford progeria syndrome," Rejuvenation Research, vol. 15, no. 2, pp. 123-127, 2012.

[20] H. Dweep, C. Sticht, P. Pandey, and N. Gretz, "MiRWalkdatabase: prediction of possible miRNA binding sites by "walking" the genes of 3 genomes," Journal of Biomedical Informatics, vol. 44, no. 5, pp. 839-847, 2011.

[21] D. Warde-Farley, S. L. Donaldson, O. Comes et al., "The GeneMANIA prediction server: biological network integration for gene prioritization and predicting gene function," Nucleic Acids Research, vol. 38, supplement 2, pp. W214-W220, 2010.

[22] M. W. Pfaffl, G. W. Horgan, and L. Dempfle, "Relative expression software tool (REST) for group-wise comparison and statistical analysis of relative expression results in real-time PCR," Nucleic acids research, vol. 30, no. 9, article e36, 2002.

[23] A. Bommarito, P. Richiusa, E. Carissimi et al., " $B R A F^{V 600 E}$ mutation, TIMP-1 upregulation, and NF- $\kappa$ B activation: closing the loop on the papillary thyroid cancer trilogy," EndocrineRelated Cancer, vol. 18, no. 6, pp. 669-685, 2011.

[24] A. Criscimanna, G. Zito, A. Taddeo et al., "In vitro generation of pancreatic endocrine cells from human adult fibroblast-like limbal stem cells," Cell Transplantation, vol. 21, no. 1, pp. 73-90, 2012.

[25] E. Doxakis, "Principles of miRNA-target regulation in metazoan models," International Journal of Molecular Sciences, vol. 14, no. 8, pp. 16280-16302, 2013.

[26] C.-P. Lin, Y. J. Choi, G. G. Hicks, and L. He, “The emerging functions of the p53-miRNA network in stem cell biology," Cell Cycle, vol. 11, no. 11, pp. 2063-2072, 2012.

[27] D. M. Popović, "Current advances in research of cytochrome c oxidase," Amino Acids, vol. 45, no. 5, pp. 1073-1087, 2013.

[28] R. G. Pestell, "New roles of cyclin D1," American Journal of Pathology, vol. 183, no. 1, pp. 3-9, 2013.

[29] E. Choi and K. C. Hwang, "MicroRNAs as novel regulators of stem cell fate," World Journal of Stem Cells, vol. 5, no. 4, pp. 172187, 2013.

[30] J. Jia, P. Yao, A. Arif, and P. L. Fox, "Regulation and dysregulation of 3/UTR-mediated translational control," Current Opinion in Genetics and Development, vol. 23, no. 1, pp. 29-34, 2013.

[31] J. T. Huang, J. Wang, V. Srivastava, S. Sen, and S. M. Liu, "MicroRNA machinery genes as novel biomarkers for cancer," Frontiers in Oncology, vol. 4, article 113, 2014.

[32] P. A. Muller and K. H. Vousden, "Mutant p53 in cancer: new functions and therapeutic opportunities," Cancer Cell, vol. 25, no. 3, pp. 304-317, 2014.

[33] V. G. Antico Arciuch, M. A. Russo, M. Dima et al., "Thyrocytespecific inactivation of $\mathrm{p} 53$ and Pten results in anaplastic thyroid carcinomas faithfully recapitulating human tumors," Oncotarget, vol. 2, no. 12, pp. 1109-1126, 2011. 
[34] L. Boominathan, "The tumor suppressors p53, p63, and p73 are regulators of microRNA processing complex," PLOS ONE, vol. 5, no. 5, Article ID e10615, 2010.

[35] Z. Yu, L. Wang, C. Wang et al., "Cyclin D1 induction of dicer governs microRNA processing and expression in breast cancer," Nature Communications, vol. 4, article 2812, 2013.

[36] X. Xu, S. Duan, F. Yi, A. Ocampo, G.-H. Liu, and J. C. Izpisua Belmonte, "Mitochondrial regulation in pluripotent stem cells," Cell Metabolism, vol. 18, no. 3, pp. 325-332, 2013. 


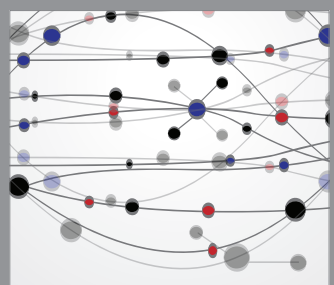

The Scientific World Journal
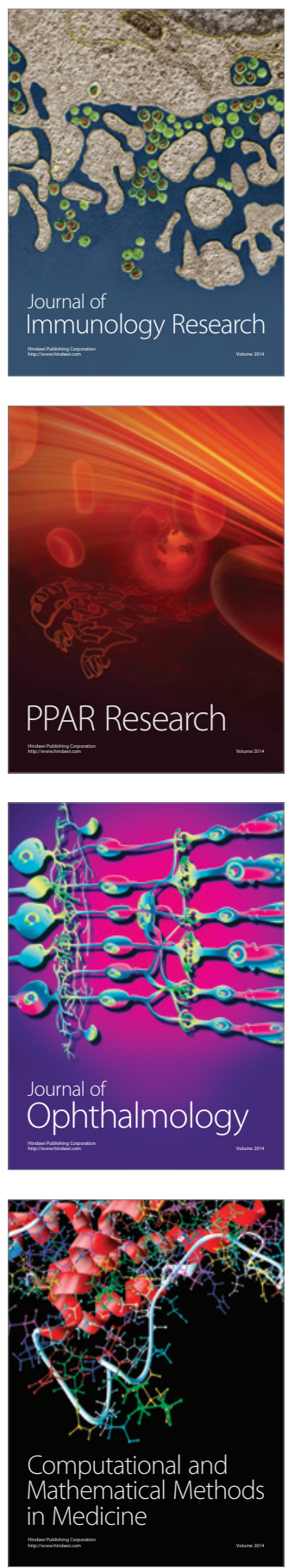

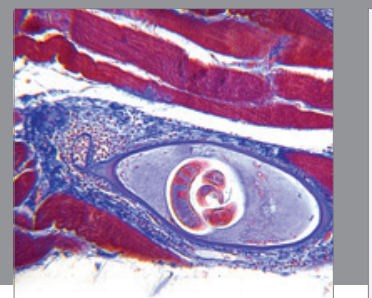

Gastroenterology

Research and Practice
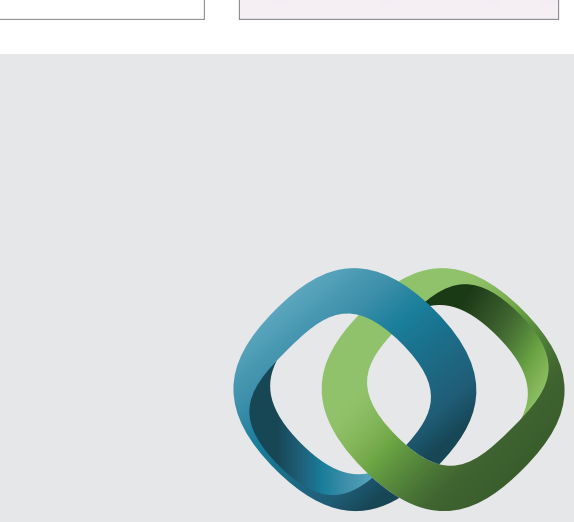

\section{Hindawi}

Submit your manuscripts at

http://www.hindawi.com
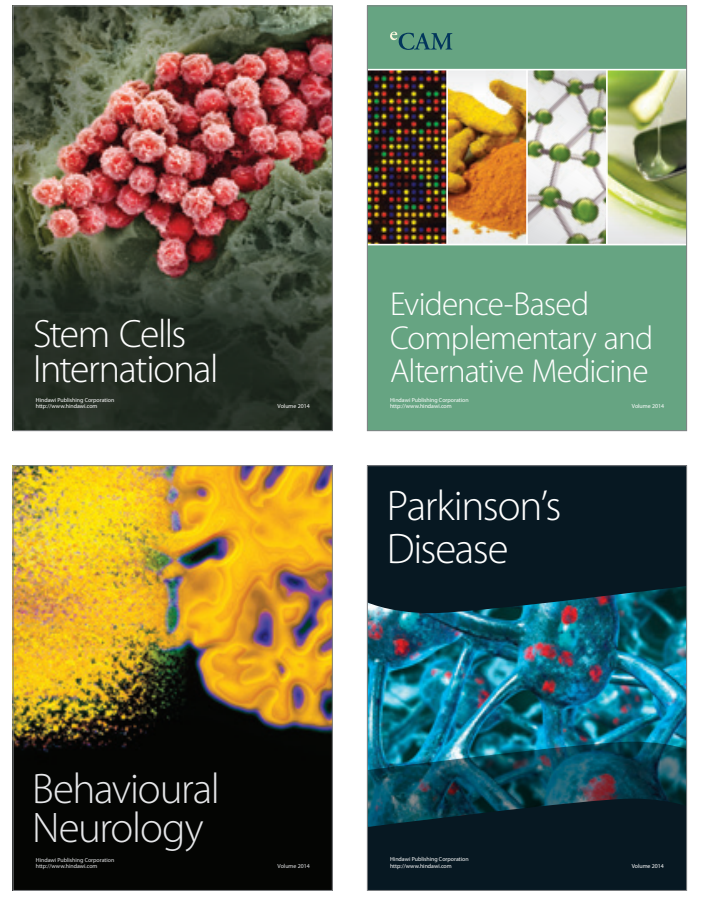
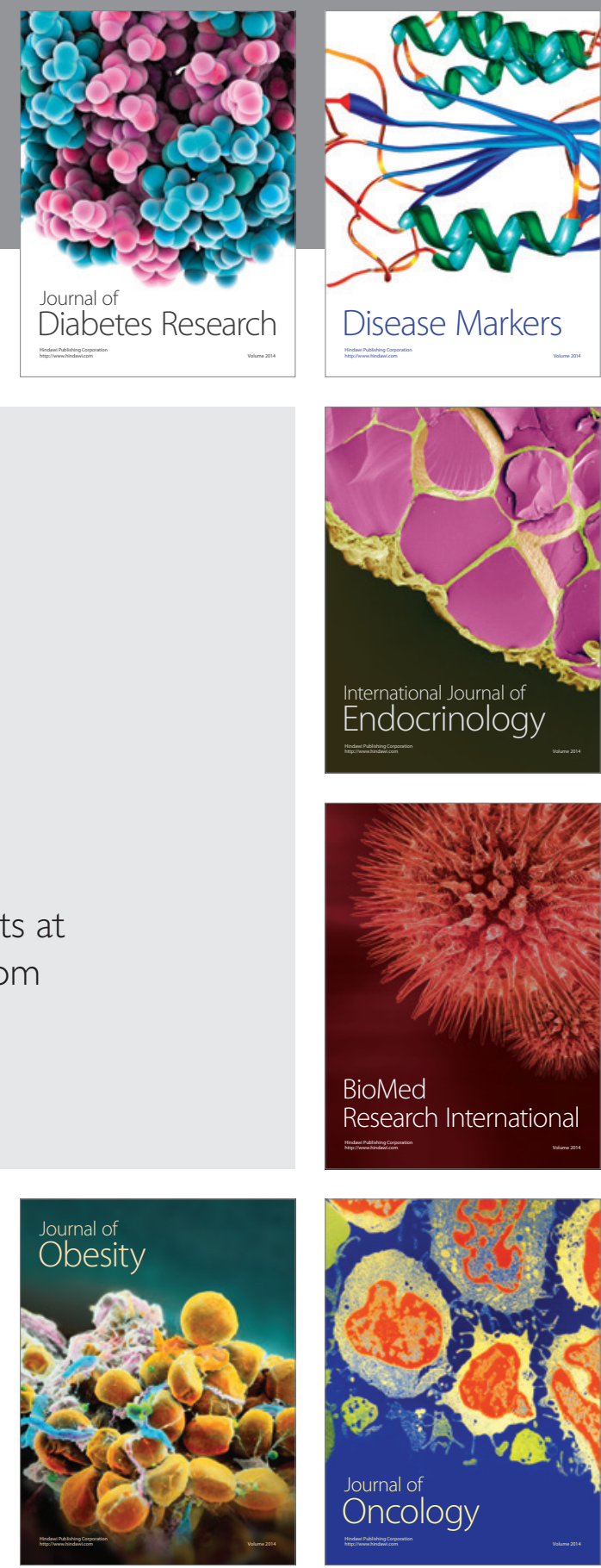

Disease Markers
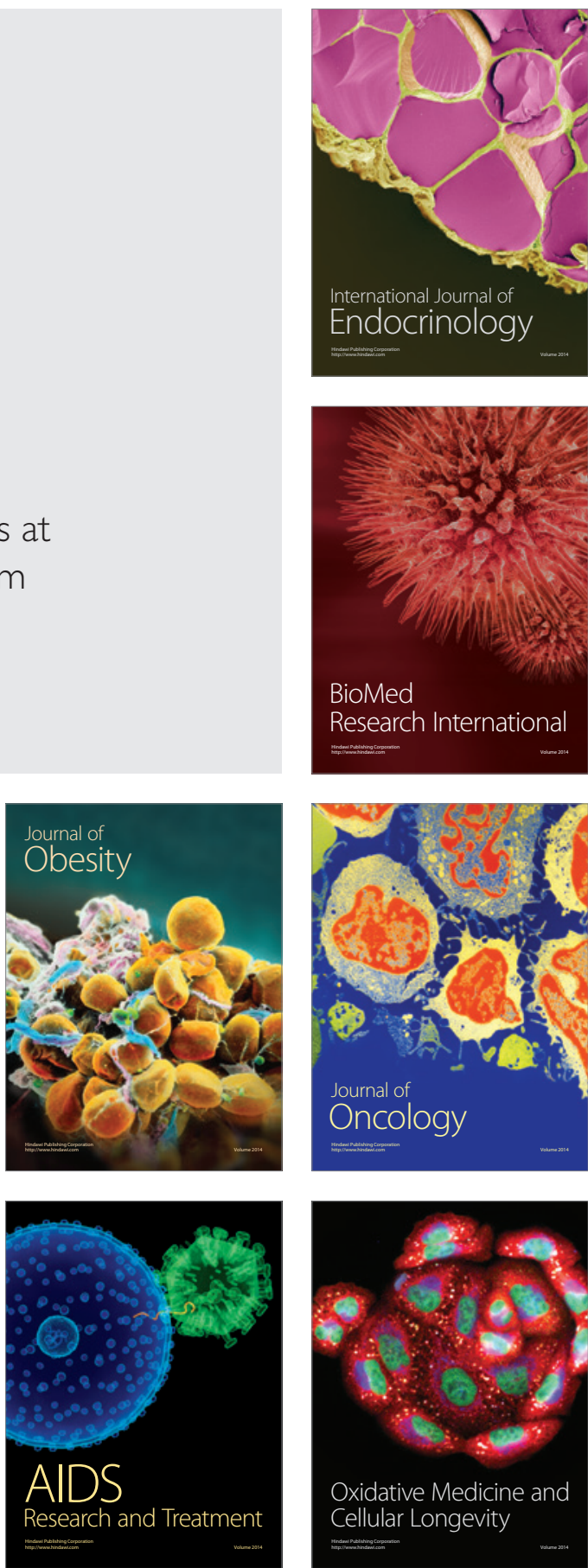